\title{
Optimized signal-to-noise ratio with shot noise limited detection in Stimulated Raman Scattering microscopy
}

M. J. B. Moester

F. Ariese

J. F. de Boer

jfdeboer@few.vu.nl

\author{
Institute for Lasers, Life and Biophotonics Amsterdam, Department of Physics and Astronomy, VU \\ University Amsterdam, The Netherlands \\ Institute for Lasers, Life and Biophotonics Amsterdam, Department of Physics and Astronomy, VU \\ University Amsterdam, The Netherlands \\ Indian Institute of Science, Department of Inorganic and Physical Chemistry, Bangalore 560012, India \\ Institute for Lasers, Life and Biophotonics Amsterdam, Department of Physics and Astronomy, VU \\ University Amsterdam, The Netherlands
}

We describe our set-up for Stimulated Raman Scattering (SRS) microscopy with shot noise limited detection for a broad window of biologically relevant laser powers. This set-up is used to demonstrate that the highest signal-to-noise ratio (SNR) in SRS with shot noise limited detection is achieved with a time-averaged laser power ratio of 1:2 of the unmodulated and modulated beam. In SRS, two different coloured laser beams are incident on a sample. If the energy difference between them matches a molecular vibration of a molecule, energy can be transferred from one beam to the other. By applying amplitude modulation to one of the beams, the modulation transfer to the other beam can be measured. The efficiency of this process is a direct measure for the number of molecules of interest in the focal volume. Combined with laser scanning microscopy, this technique allows for fast and sensitive imaging with sub-micrometre resolution. Recent technological advances have resulted in an improvement of the sensitivity of SRS applications, but few show shot noise limited detection.

The dominant noise source in this SRS microscope is the shot noise of the unmodulated, detected beam. Under the assumption that photodamage is linear with the total laser power, the optimal SNR shifts away from equal beam powers, where the most signal is generated, to a 1:2 power ratio. Under these conditions the SNR is maximized and the total laser power that could induce photodamage is minimized. Compared to using a 1:1 laser power ratio, we show improved image quality and a signal-to-noise ratio improvement of $8 \%$ in polystyrene beads and C. Elegans worms. Including a non-linear damage mechanism in the analysis, we find that the optimal power ratio converges to a 1:1 ratio with increasing order of the non-linear damage mechanism.

[DOI: http://dx.doi.org/10.2971/jeos.2015.15022]

Keywords: Nonlinear Raman microscopy, shot noise limited detection, signal-to-noise ratio

\section{INTRODUCTION}

In recent years, coherent Raman scattering microscopy has found many applications in biomedical imaging. This group of sensitive imaging techniques based on intrinsic chemical contrast has advantages over fluorescence microscopy, mainly because it is a label-free technique. This allows imaging of small molecules or easily disturbed systems without perturbing their biological function [1]-[3]. In particular Stimulated Raman Scattering (SRS) microscopy has shown great promise in this field. Technological developments have improved sensitivity [4]-[6] and imaging speed [7, 8] and brought this from a concept to a promising technique for biomedical imaging [9].

In SRS, two different coloured laser beams are incident on a sample. If the energy difference between them matches a molecular vibration of a molecule, energy can be transferred from one beam to the other. By applying amplitude modulation to one of the beams, the modulation transfer to the other beam can be measured. The efficiency of this process is a direct measure for the number of molecules of interest in the focal volume. Combined with laser scanning microscopy, this technique allows for fast and sensitive imaging with submicrometre resolution [1].
The first demonstrations of SRS microscopy showed poor detection limits, requiring many thousands of molecules with large Raman scattering cross sections in the focal volume. Improvements in the detection of the small signals have lowered the limit of detection. By resonant amplification of the signal [10], using chemical labelling [11, 12], or implementing a different detection geometry $[5,6,13]$, the sensitivity can be pushed close to the shot noise limit. This has allowed imaging not only in the $\mathrm{CH}$-stretch region but also in the fingerprint region, where Raman signals are often weaker [14].

Because signal levels in SRS microscopy are measured as a small change on a high background intensity, care must be taken to optimize the imaging parameters to achieve the best signal-to-noise ratio (SNR) for the applied laser power. The best attainable situation is realized when shot noise dominates the noise contributions, because the shot noise is an intrinsic property of the detected light.

In this paper, we show shot noise limited detection for a broad range of laser powers with an experimental set-up based mainly on commercial components. This system is used to demonstrate the validity of the theoretical prediction that 
the highest SNR can be achieved with a time-averaged laser power ratio of 1:2 of the unmodulated and modulated beams. An SNR improvement of $9 \%$ is attainable compared to the more commonly used 1:1 laser power ratio, without any changes to the sample or set-up. While small, this increase can enhance the image quality without sacrificing signal by simply tuning the laser power appropriately.

\section{THEORY}

The amount of signal generated in SRS is described by

$$
S \propto[c] \sigma I_{\text {pump }} I_{\text {Stokes }}
$$

where $[c]$ is the concentration of scatterers, $\sigma$ the scattering cross section and $I_{\text {pump }}$ and $I_{\text {Stokes }}$ the laser power densities of the pump and Stokes beam, respectively $[6,15]$. For picosecond SRS, the power density of both beams is linear with the laser intensity, as the pulse duration and focusing conditions are the same. In Stimulated Raman Loss (SRL), the amplitude of the Stokes beam is modulated to provide the frequency for lock-in detection. The time-averaged intensity of this modulated beam is half the intensity during 'on' time. The total laser power density $I_{\text {total }}$ is defined as the sum of $I_{\text {pump }}$ and $I_{\text {Stokes }}$;

$$
I_{\text {total }}=I_{\text {pump }}+\frac{1}{2} I_{\text {Stokes }}
$$

In any application, the photodamage caused to the sample sets an upper limit on the total power applied. In our set-up, both $I_{\text {pump }}$ and $I_{\text {Stokes }}$ have an 8 picosecond pulse length and wavelengths in the near IR, therefore mechanisms of photodamage are expected to be similar for both colours [16]. In the case of a linear dependence of photodamage on the total power, any division of power between the two beams is assumed to result in the same amount of exposure and photodamage. The distribution of this total power between the two colours determines the amount of SRS signal and the resulting SNR, and should be carefully considered.

By combining Eqs. (1) and (2) and evaluating the derivative as a function of $I_{\text {pump }}$, it is found that the maximum signal is obtained when the time-averaged intensities of pump and Stokes beam are equal. This is also the optimal experimental choice when the noise is independent of the laser powers in non-shot noise limited implementations and for related coherent Raman methods where the signal is measured against a dark background, such as in Coherent AntiStokes Raman Scattering (CARS) and Raman Induced Kerr Effect Spectroscopy (RIKES). However, when the dominant noise source is shot noise, as in our implementation of SRS microscopy, the noise level only depends on the intensity of the detected beam and the SNR is given by

$$
S N R \propto \frac{S}{\sqrt{I_{\text {pump }}}}
$$

The maximum SNR is found when the derivative of Eq. (3) with respect to $I_{\text {pump }}$ is equal to zero, resulting in a ratio of 1:2 of the time-averaged intensities of the pump and Stokes beams. In this case, the peak power of the Stokes beam during the 'on' phase of the modulation is four times that of the pump beam. This 1:2 power ratio would result in a theoretical improvement of the SNR of $9 \%$ compared to a 1:1 power ratio. The same principle applies to Stimulated Raman Gain (SRG), but in that case the ratio is reversed because the Stokes beam is detected. The validity of this argument also extends to other coherent Raman methods where the signal is measured as an increase of the detected beam, such as optically heterodyne detected RIKES [17] and heterodyne CARS [18]. Here the time-averaged intensity of the detected, unmodulated beam should also be half the intensity of the modulated beam to realize the highest SNR.

\section{EXPERIMENTAL SET-UP}

We have built a flexible imaging set-up for Stimulated Raman Loss microscopy (Figure 1(a)). An $80 \mathrm{MHz}$ laser (Lumera Plecter Duo) with 8 ps pulses at $532 \mathrm{~nm}$ is used to pump an Optical Parametric Oscillator (OPO) (APE Levante Emerald) with a wavelength output range of $775-990 \mathrm{~nm}$. This output is overlapped in space and time with the $1064 \mathrm{~nm}$ fundamental laser line. A combination of a half-wave plate and a polarizing beamsplitter in each beam is used to control the polarization state and laser intensity. An acousto-optic modulator (Crystal Technology 3080194) is used for $3.636 \mathrm{MHz}$ sinusoidal intensity modulation of the $1064 \mathrm{~nm}$ Stokes beam. A Zeiss laser scanning microscope (type LSM 7MP) with $32 \times$ objective (C-Achroplan $\mathrm{W}, \mathrm{NA}=0.85$ ) is used to image samples with non-descanned detection in forward scattering mode. Optical filters reject the $1064 \mathrm{~nm}$ modulated Stokes beam and the pump beam light is collected on a Si photodiode (Thorlabs DET36A). The signal is amplified with a homebuilt transimpedance amplifier (using a Texas Instruments OPA656) before demodulation in a lock-in amplifier (Stanford Research Systems SR844). The amplifier generates an $86 \mathrm{~dB}$ transimpedance gain on the photodiode current and includes a $3.636 \mathrm{MHz}$ bandpass filter.

Shot noise limited detection is achieved when the shot noise is equal to or larger than any other noise source. In our case, at the lower end, this is at $75 \mu \mathrm{W}$ of pump power on the detector (Figure 1(b)), where the shot noise is equal to the measured thermal or Johnson-Nyquist noise of $71 \mathrm{nV} / \sqrt{\mathrm{Hz}}$. The upper limit of $10 \mathrm{~mW}$ is defined by the saturation current of the photodiode ( $5 \mathrm{~mA})$ which is well before the relative intensity noise of the laser becomes significant. The effective shot noise window with a collection efficiency of $50 \%$ and a $1: 2$ power ratio is from $0.45-60 \mathrm{~mW}$ of total power on the sample, which corresponds well with biologically acceptable intensities.

\section{RESULTS AND DISCUSSION}

We have experimentally validated the predicted optimal ratio between $I_{\text {pump }}$ and $I_{\text {Stokes }}$. A sample of $2 \mu \mathrm{m}$ polystyrene beads in water was sandwiched between a glass slide and a coverslip. SRL images were taken at the $2845 \mathrm{~cm}^{-1} \mathrm{CH}_{2}$ stretch vibration. Figure 2 shows the background level, signal intensity and SNR for different ratios of pump and Stokes power at a total power of $9 \mathrm{~mW}$ on the sample. The highest SNR was found for a ratio of 1:2, which matches the theoretical prediction.

The SNR here is defined as the measured signal divided by 


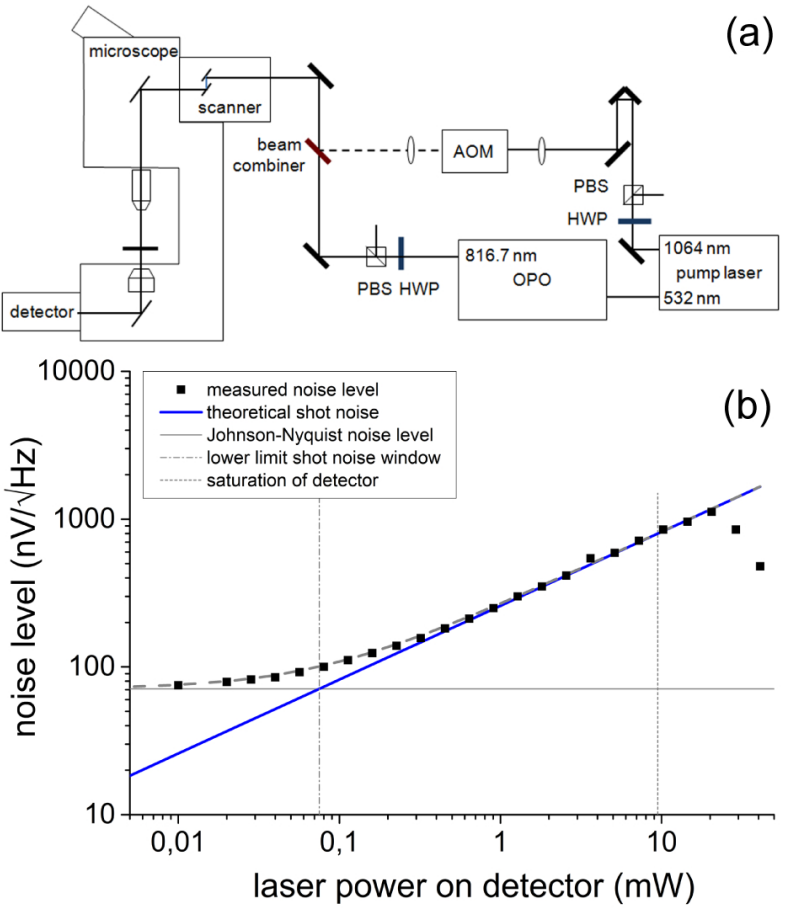

FIG. 1 (a) Experimental set-up for SRS microscopy, showing the pump beam at $816.7 \mathrm{~nm}$ and the Stokes beam at $1064 \mathrm{~nm}$. PBS: polarizing beamsplitter, HWP: half-wave plate, AOM: acousto-optic modulator; (b) Noise characteristics of the detection system as a function of $I_{\text {pump }}$ on the detector. Measured data points (black squares) match the sum of theoretical shot noise (blue line) and measured electronic noise (JohnsonNyquist noise level, grey line). Shot noise limited detection is achieved from $75 \mu \mathrm{W}$ to $10 \mathrm{~mW}$ on the detector.

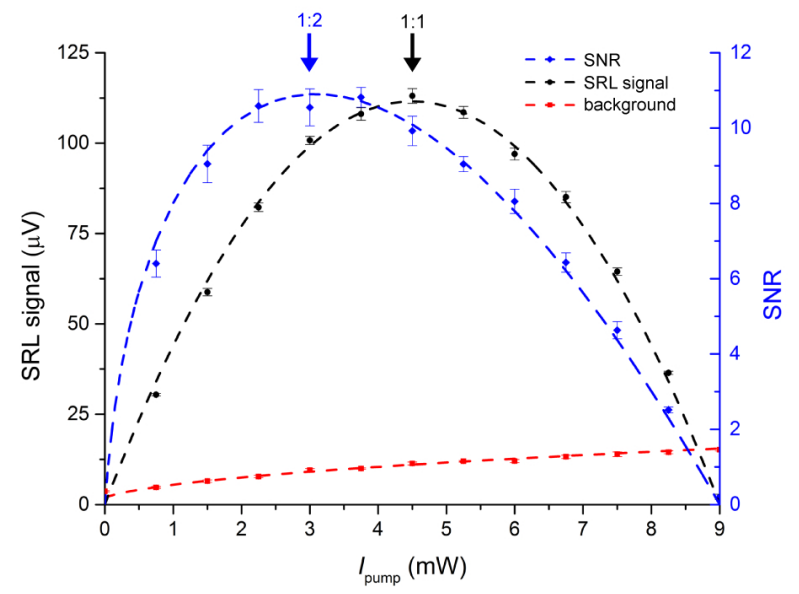

FIG. 2 The SRL signal intensity (black circles, left $y$-axis), background intensity (red squares, left $y$-axis) and SNR (blue diamonds, right y-axis) as a function of the $I_{\text {pump }}$ intensity. Intensities were measured at six regions of interest of $\phi_{2} \mu \mathrm{m}$ on and off beads; error bars represent one standard deviation of the mean value of six regions. The total laser intensity on the sample is constant at $9 \mathrm{~mW}$. $I_{\text {pump }}: I_{\text {Stokes }}$ ratios of 1:2 and $1: 1$ are indicated.

the sum of the noise contributions, which consist of electronic noise and shot noise. At the laser powers used, the relative intensity noise of the laser at this modulation frequency is very small and does not contribute. The background level (the amplitude measured at a location where no Raman vibration is accessible) is equal to the sum of the constant electronic noise and the shot noise and consistent with the theory (grey dashed line in Figure 1(b)). Other contributions that could have been expected are related to non-resonant background caused by the Raman-induced refractive index change (Kerr effect). As the measured background correlates exactly to the theoretical shot noise, these effects are negligible here. This is reasonable because the large Raman cross-section of the $\mathrm{CH}_{2}$-stretch allows low laser powers to be used. Furthermore, the nonresonant medium is spatially uniform, eliminating the main source of Raman-induced refractive index changes [19].

Data points were measured as the mean intensity of six regions of interest of $\varnothing 1 \mu \mathrm{m}$ in the images on and off the beads for signal and background respectively. Error bars represent one standard deviation of the mean value of those regions. The dashed line through the background data points is the sum of measured electronic noise and theoretical shot noise. The fitted line through the signal data points is based on the theory described above, with the only free parameter being the amplitude of the curve. This is a function of concentration and scattering cross section and includes the detection parameters (collection efficiency, spectral sensitivity of the detector, transimpedance gain). This amplitude, combined with the theory described above, is used to determine the dashed line through the SNR data points. The measured electronic noise is included in the predicted SNR by adding it to the shot noise in the denominator of Eq. (3). This primarily has an effect for low pump powers, as at a 1:2 ratio, the shot noise is already 4.5 times as large as the electronic noise. In this configuration, with the current collection efficiency and applied maximal power, the maximal SNR was found for a 1:1.9 ratio, so the effect of the electronic noise is minimal. The contribution of electronic noise lowers the maximum improvement in SNR from $9 \%$ to $8 \%$ compared to using a 1:1 laser power ratio. This improvement is relative to the same set-up, changing only the laser powers of the excitation beams. While the enhancement is small, it does not require any new hardware and can be implemented on any system.

The image quality for four different laser power ratios is demonstrated in Figure 3. Although the intensity of the $2 \mu \mathrm{m}$ polystyrene beads is highest at equal laser power, the background is also stronger. The SNR at a 1:2 ratio was higher than at a 1:1 ratio, as predicted. Images were recorded at $256 \times 256$ pixels with a pixel dwell time of $100 \mu \mathrm{s}$. Four frames were averaged for each image, total time per image was 36 seconds.

The time constant of the lock-in amplifier was set to $100 \mu \mathrm{s}$ with a slope of $24 \mathrm{~dB} /$ oct. The amplitude output at a sensitivity of $300 \mu \mathrm{V}$ was supplied to the ZEN microscopy software, which was used to record the images. This robust configuration was chosen as it required no prior calibration of the lock-in phase. Though experimentally more complex, measurement of the phase-sensitive X-component could have further improved the SNR by a factor of $\sqrt{2}$. The lock-in amplifier locks to the highest amplitude at the provided reference frequency, which is correct for the SRS signal, but when no signal is present, it results in the maximum magnitude of the shot noise, rather than only the in-phase component. The expected improvement would be proportional to the SNR and does not change the relative improvement of using a 1:2 laser power ratio compared to a 1:1 ratio. 

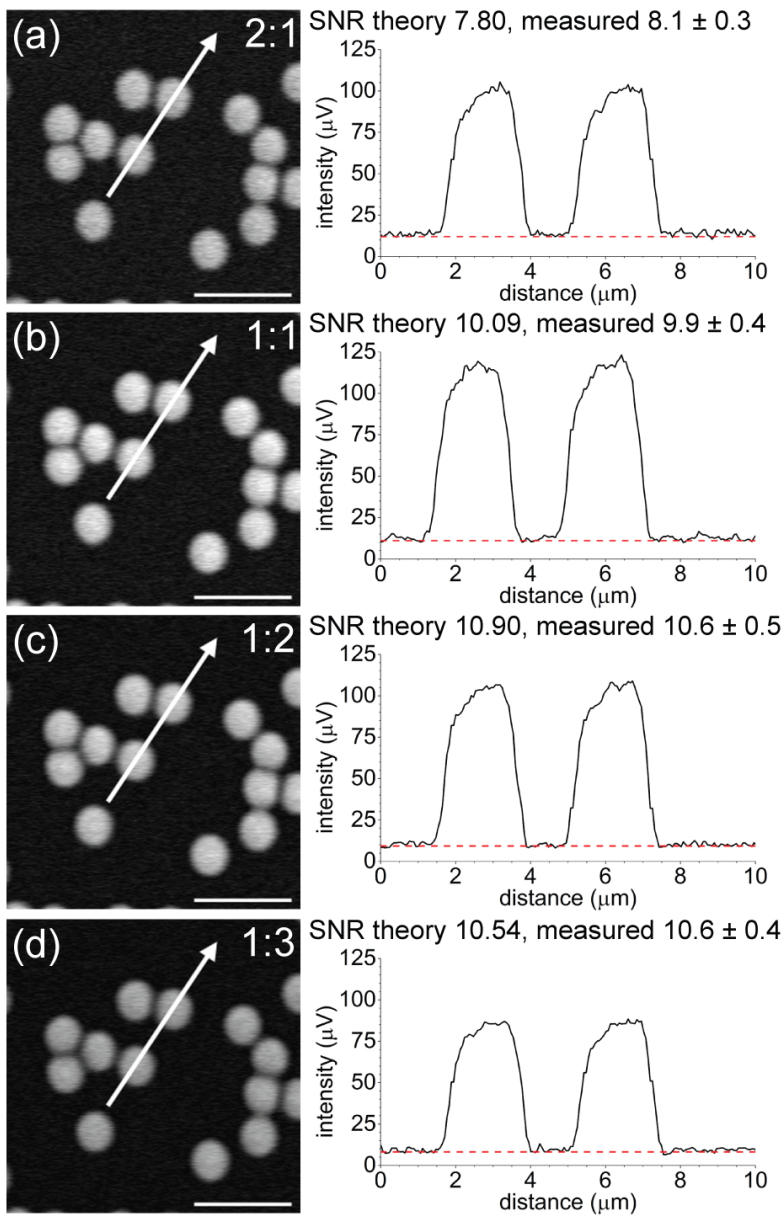

FIG. 3 Microscopy images at different power ratios of $I_{\text {pump }}$ to $I_{\text {Stokes }}$ with a constant total power. (a-d) SRL images at $2845 \mathrm{~cm}^{-1}$ on $2 \mu \mathrm{m}$ polystyrene beads showing the imaging quality at 2:1, 1:1, 1:2 and 1:3 $I_{\text {pump }}: I_{\text {Stokes }}$ laser power ratio. Total power on the sample was $9 \mathrm{~mW}$. Cross sectional traces along the arrow are shown adjacent. scale bar is $5 \mu \mathrm{m}$.

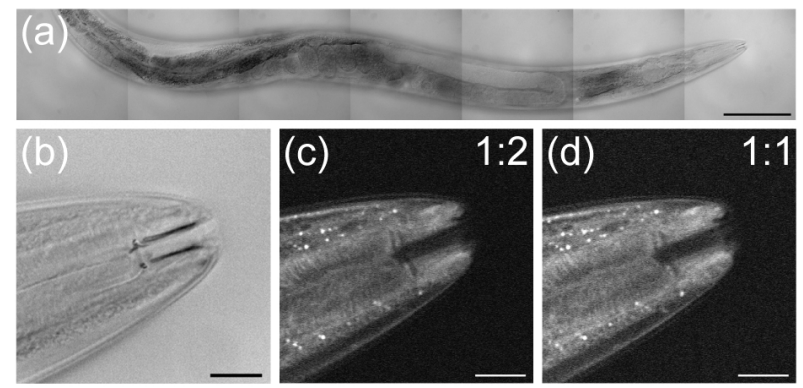

FIG. 4 Microscopy images of the head end of an adult C. Elegans roundworm. (a) White light camera image, scale bar is $100 \mu \mathrm{m}$; (b) Detail of (a) at the worm mouth, scale bar is $10 \mu \mathrm{m}$; (c-d) SRL images at $2845 \mathrm{~cm}^{-1}$ at $I_{\text {pump }}: I_{\text {Stokes }}$ ratios of $1: 2$ and 1:1 respectively. Total power on the sample was constant at $9 \mathrm{~mW}$. The SNR improvement is $8 \pm 2 \%$. Scale bar is $10 \mu \mathrm{m}$.

The SNR improvement of $8 \%$ is small, but the effect could be important at lower signal levels, as are encountered in real biological samples. In Figure 4 the results of imaging the head end of an adult C. Elegans roundworm at $2845 \mathrm{~cm}^{-1}$ are shown. Mature Bristol N2 wildtype animals were anaesthetized with $5 \mathrm{mM}$ levamisole dissolved in M9 buffer and mounted on a thin agarose film on a glass slide and covered with a coverslip. The SRL signal originates from $\mathrm{CH}_{2}$ - stretches in the cellular membranes and the intracellular lipid droplets that are abundant in these animals [20]. Images shown are single recordings of $1024 \times 1024$ pixels with a pixel dwell time of $100 \mu \mathrm{s}$. The time constant of the lock-in amplifier was set to $100 \mu$ s with a slope of $24 \mathrm{~dB} /$ oct and $300 \mu \mathrm{V}$ sensitivity. The improvement in SNR was calculated from six $\varnothing 3 \mu \mathrm{m}$ regions of interest on the worm compared to the background outside the worm body. An average improvement in the SNR of $8 \pm 2 \%$ was found for a 1:2 power ratio compared to a 1:1 ratio. Even though the $\mathrm{CH}_{2}$-stretch has a large Raman cross-section, the signal level is still low at regions of low concentration. The improvement in SNR results in an improvement of contrast compared to the background. In Figure 4, this effect can be appreciated from the enhanced contrast in regions of low signal, such as at the outer membranes of the worm.

We have shown that the optimal laser power ratio for shot noise limited applications of SRS is a 1:2 ratio of the unmodulated and modulated beams, for the situation with a given maximum combined average power of pump and Stokes laser beams. In the case of a non-linear damage mechanism, not just the total time-averaged power defines the amount of damage, but the time-dependent intensity profile of the modulated beam becomes important. The amount of damage is described as proportional to the intensity of the applied laser power, raised to the power of the damage order:

$$
D \propto I(t)^{n}
$$

Where $D$ symbolizes the amount of damage, $I(t)$ the intensity profile in time and $n$ is the order of the damage mechanism [21]. The value of $n$ has been determined for the typical laser parameters of power, wavelength, pulse length and repetition rate used in coherent Raman scattering microscopy. For dense structures such as protein clusters, it is near 1, while for a less dense environment such as a cell, values of $n$ above 2 are found [16, 22]. Two types of modulation profile are commonly used in SRS; sinusoidal modulation and top hat modulation (inset Figure 5). The damage caused by these modulation profiles can be expressed as:

$$
\begin{aligned}
D_{\text {sin }} & =\left\langle\left[I_{\text {pump }}+\frac{1}{2}\left(1+\sin (\omega t) I_{\text {Stokes }}\right)\right]^{n}\right\rangle \\
D_{\text {tophat }} & =\frac{1}{2}\left[I_{\text {pump }}\right]^{n}+\frac{1}{2}\left[I_{\text {pump }}+I_{\text {Stokes }}\right]^{n}
\end{aligned}
$$

Here, $\omega$ is the modulation frequency and the chevrons indicate a time average over an integer number of modulation cycles. The time averaging is included in the top hat modulation profile by the factors of $\frac{1}{2}$. The same signal level is achieved with these different modulation profiles, but the higher instantaneous peak powers of top hat modulation will result in more damage to the sample for non-linear damage mechanisms. This indicates the choice for a sinusoidal modulation profile is usually beneficial.

We evaluated the optimum by finding the ratio giving the highest SNR for a constant amount of damage for these modulation profiles. Figure 5 shows this optimal ratio of $I_{\text {pump }}$ to $I_{\text {Stokes }}$ as a function of the order of the damage mechanism. The optimal ratio is independent of pixel dwell time and total amount of damage. For a value of $n=1$, we find an optimal 


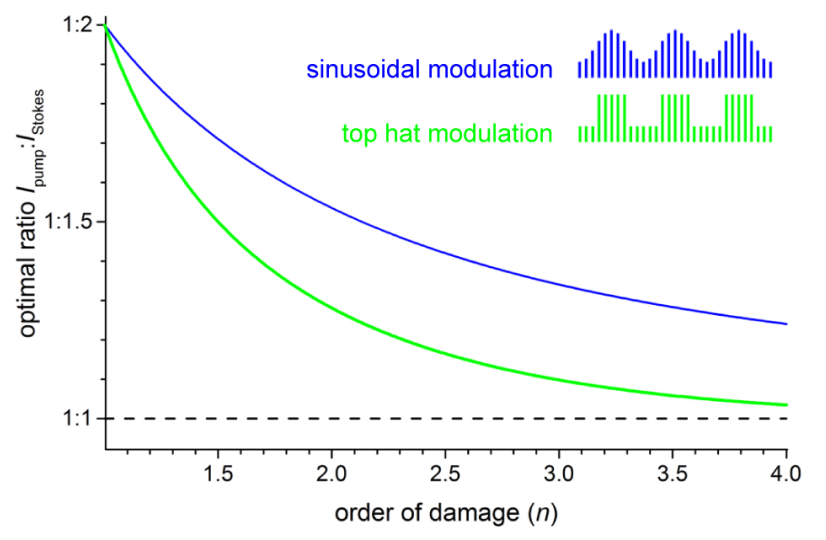

FIG. 5 The calculated optimal ratio of $I_{\text {pump }}$ to $I_{\text {Stokes }}$ yielding the highest SNR at a constant damage for a sinusoidal (blue line) and a top hat (red line) modulation profile as a function of the order of damage $(n)$. The inset shows a graphical representation of the modulation profiles. For linear damage $(n=1)$, the optimal ratio is $1: 2$, for higher values of $n$, both modulation profiles converge toward a 1:1 ratio.

ratio of 1:2, but for higher values of $n$ the optimum shifts towards a 1:1 ratio.

To find the optimal ratio of $I_{\text {pump }}$ to $I_{\text {Stokes }}$ for an application, one would ideally need to know the damage mechanism of the sample to evaluate the equations shown above. In practice, other considerations could also play a role in determining the choice of laser powers, such as a different damage mechanism for the two colours, saturation of the detection system limiting the detected power [13] or a limitation in the available power of one or both colours. In such cases, the theory in Section 2 and above should be evaluated to achieve the maximum SNR within the limitations of the experimental system.

\section{CONCLUSIONS}

We have shown shot noise limited detection over a broad window of biologically relevant laser powers. Our version of shot noise limited detection is easy to implement, with mostly commercial components and a homebuilt amplifier. A shot noise limited detection window is achieved from $0.45-60 \mathrm{~mW}$ of total power on the sample. These laser powers are a useful range for imaging of biological samples.

We have experimentally confirmed that the best SNR is achieved when the modulated beam has double the timeaveraged intensity of the detected, unmodulated beam for a linear photodamage mechanism. Particularly in applications where small signals need to be distinguished from a dark background, shot noise limited detection should be implemented and the SNR can be maximized using a $I_{\text {pump }}: I_{\text {Stokes }}$ ratio of 1:2. For non-linear photodamage, the optimal ratio converges to a 1:1 ratio with increasing order of the non-linear damage mechanism.

\section{ACKN OWLEDGEMENTS}

The authors would like to acknowledge $\mathrm{H}$. Voet and A. van der Gracht for their help with the development of the transimpedance amplifier, L. Zada for assistance on the setup and experiments, J. Mijalkovic for the C. Elegans samples and D. Boonzajer Flaes for help with modelling the intensity profiles. We gratefully acknowledge financial support from LaserLaB Amsterdam, Laserlab-Europe (EC-GA 284464), and the Netherlands Organisation for Scientific Research (NWO) with a Vici (JFdB) and an NWO-Groot grant (JFdB).

\section{References}

[1] C. W. Freudiger, W. Min, B. G. Saar, S. Lu, G. R. Holtom, C. W. He, J. C. Tsai, J. X. Kang, and X. S. Xie, "Label-Free Biomedical Imaging with High Sensitivity by Stimulated Raman Scattering Microscopy," Science 322, 1857-1861 (2008).

[2] P. Nandakumar, A. Kovalev, and A. Volkmer, "Vibrational imaging based on stimulated Raman scattering microscopy," New J. Phys. 11, 9 (2009).

[3] T. Meyer, M. Schmitt, B. Dietzek, and J. Popp, "Accumulating advantages, reducing limitations: Multimodal nonlinear imaging in biomedical sciences - the synergy of multiple contrast mechanisms," J. Biophotonics 6, 887-904 (2013).

[4] Y. Ozeki, Y. Kitagawa, K. Sumimura, N. Nishizawa, W. Umemura, S. Kajiyama, K. Fukui, and K. Itoh, "Stimulated Raman scattering microscope with shot noise limited sensitivity using subharmonically synchronized laser pulses," Opt. Express 18, 13708-13719 (2010).

[5] K. Nose, Y. Ozeki, T. Kishi, K. Sumimura, N. Nishizawa, K. Fukui, Y. Kanematsu, and K. Itoh, "Sensitivity enhancement of fiber-laserbased stimulated Raman scattering microscopy by collinear balanced detection technique," Opt. Express 20, 13958-13965 (2012).

[6] C. W. Freudiger, W. L. Yang, G. R. Holtom, N. Peyghambarian, X. S. Xie, and K. Q. Kieu, "Stimulated Raman scattering microscopy with a robust fibre laser source," Nat. Photonics 8, 153-159 (2014).

[7] B. G. Saar, C. W. Freudiger, J. Reichman, C. M. Stanley, C. R. Holtom, and X. S. Xie, "Video-Rate Molecular Imaging in Vivo with Stimulated Raman Scattering," Science 330, 1368-1370 (2010).

[8] Y. Ozeki, W. Umemura, Y. Otsuka, S. Satoh, H. Hashimoto, K. Sumimura, N. Nishizawa, K. Fukui, and K. Itoh, "High-speed molecular spectral imaging of tissue with stimulated Raman scattering," Nat. Photonics 6, 844-850 (2012).

[9] C. W. Freudiger, R. Pfannl, D. A. Orringer, B. G. Saar, M. B. Ji, Q. Zeng, L. Ottoboni, W. Ying, C. Waeber, J. R. Sims, P. L. De Jager, O. Sagher, M. A. Philbert, X. Y. Xu, S. Kesari, X. S. Xie, and G. S. Young, "Multicolored stain-free histopathology with coherent Raman imaging," Lab. Invest. 92, 1492-1502 (2012).

[10] M. N. Slipchenko, R. A. Oglesbee, D. L. Zhang, W. Wu, and J. X. Cheng, "Heterodyne detected nonlinear optical imaging in a lock-in free manner," J. Biophotonics 5, 801-807 (2012).

[11] S. Hong, T. Chen, Y. Zhu, A. Li, Y. Huang, and X. Chen, "Live-Cell Stimulated Raman Scattering Imaging of Alkyne-Tagged Biomolecules," Angew. Chem. Int. Edit. 53, 5827-5831 (2014).

[12] L. Wei, F. Hu, Y. Shen, Z. Chen, Y. Yu, C.-C. Lin, M. C. Wang, and W. Min, "Live-cell imaging of alkyne-tagged small biomolecules by stimulated Raman scattering," Nat. Methods 11, 410-2 (2014).

[13] W. Rock, M. Bonn, and S. H. Parekh, "Near shot-noise limited hyperspectral stimulated Raman scattering spectroscopy using low energy lasers and a fast CMOS array," Opt. Express 21, 15113-15120 (2013). 
[14] X. Zhang, M. B. J. Roeffaers, S. Basu, J. R. Daniele, D. Fu, C. W. Freudiger, G. R. Holtom, and X. S. Xie, "Label-Free Live-Cell Imaging of Nucleic Acids Using Stimulated Raman Scattering Microscopy," Chemphyschem 13, 1054-1059 (2012).

[15] Y. Ozeki, F. Dake, S. Kajiyama, K. Fukui, and K. Itoh, "Analysis and experimental assessment of the sensitivity of stimulated Raman scattering microscopy," Opt. Express 17, 3651-3658 (2009).

[16] Y. Fu, H. F. Wang, R. Y. Shi, and J. X. Cheng, "Characterization of photodamage in coherent anti-Stokes Raman scattering microscopy," Opt. Express 14, 3942-3951 (2006).

[17] C. W. Freudiger, M. B. J. Roeffaers, X. Zhang, B. G. Saar, W. Min, and X. S. Xie, "Optical Heterodyne-Detected Raman-Induced Kerr Effect (OHD-RIKE) Microscopy," J. Phys. Chem. B 115, 5574-5581 (2011).

[18] E. O. Potma, C. L. Evans, and X. S. Xie, "Heterodyne coherent antiStokes Raman scattering (CARS) imaging," Opt. Lett. 31, 241-243 (2006).
[19] K. I. Popov, A. F. Pegoraro, A. Stolow, and L. Ramunno, "Image formation in CARS and SRS: effect of an inhomogeneous nonresonant background medium," Opt. Lett. 37, 473-475 (2012).

[20] T. Hellerer, C. Axäng, C. Brackmann, P. Hillertz, M. Pilon, and A. Enejder, "Monitoring of lipid storage in Caenorhabditis elegans using coherent anti-Stokes Raman scattering (CARS) microscopy," Proc. Natl. Acad. Sci. U S A 104, 14658-14663 (2007).

[21] A. Hopt and E. Neher, "Highly nonlinear photodamage in twophoton fluorescence microscopy," Biophys. J. 80, 2029-2036 (2001).

[22] K. König, T. W. Becker, P. Fischer, I. Riemann, and K. J. Halbhuber, "Pulse-length dependence of cellular response to intense nearinfrared laser pulses in multiphoton microscopes," Opt. Lett. 24, 113-115 (1999). 